\title{
Complementary studies on supersonic nozzle flow: Heterodyne interferometry, high-speed video shadowgraphy, and numerical simulation
}

\author{
C. D. Fredrick, T. R. Greenlee, R. W. Peterson, A. J. Schaffer, \\ K. R. Stein \& A. W. Woetzel \\ Department of Physics, Bethel University, USA
}

\begin{abstract}
Experiments and numerical simulations are carried out in the upper-level, undergraduate physics laboratory to examine the highly transient startup flow from a small nozzle. The flow is initiated by the rupture of a Mylar ${ }^{\circledR}$ diaphragm positioned between the nozzle and a pressurized air tank. The studies utilize an approach based on experimental measurements and computational methods. Experimental studies consist of dual-beam heterodyne interferometry and highspeed video (HSV) shadowgraphy. Interferometric measurements at 25 million samples per second relate optical path length changes across the nozzle flow to density variations in the flow. HSV shadowgraphy portrays the initial shock wave and compressible flow features surrounding the nozzle exit at 100,000 frames per second. Finite element computations of the unsteady, axisymmetric, compressible nozzle flow provide a field description of the flow for the full tank-nozzle-exterior domain. The studies show excellent matching between the experimental and computational methods. More importantly, the distinct strengths of the different approaches work together to provide a more comprehensive description of the nozzle flow than can achieved by the individual approaches.

Keywords: shadowgraph imaging, heterodyne interferometry, high-speed video, computational fluid dynamics, advanced undergraduate laboratory.
\end{abstract}

\section{Introduction}

There are fundamental challenges for advanced undergraduate laboratories striving to provide an engaging experience that brings together ongoing research and 
academic studies. This study of supersonic fluid flow dynamics builds naturally on computational physics, high speed video (HSV) imaging and analysis, along with quantitative gas density measurements from time-resolved interferometry. The described system has the flexibility to build on student team creativity while enhancing project-based laboratories associated with a variety of undergraduate courses - including computational methods, physical optics, and fluid dynamics.

The following sections describe in more detail the supersonic nozzle apparatus and the complementary computational, optical diagnostics, and analysis procedures that are able to fully characterize the resultant fluid flow. The flow from the nozzle and the ensuing diagnostics are initiated by a rupturing diaphragm on the pressurized tank. The flow dynamics downstream from the nozzle are categorized in three stages: a highly transient start-up flow, quasi-steady supersonic nozzle flow, and the nozzle blow-down. The experiment focuses on the transient flow from the nozzle during the initial stage, including the initial shock wave and 100-200 microseconds of supersonic flow following the shock.

Several features of this experiment are pedagogically attractive for undergraduates in physics and engineering. While the phenomenon studied is relatively simple (and very reliable) to initiate, students quickly learn to appreciate the underlying complexity of these highly transient supersonic flow patterns. Before attempting to compute and predict the expected result, HSV shadowgraphs near the nozzle (at up to 100,000 frames/sec) may serve to stimulate student interest and intuition regarding the qualitative behavior in space and time. Depending on the academic focus of the class using the system, such qualitative imaging provides an ideal background for either computational physics (numerical simulation) or time-resolved fluid density measurements from heterodyne interferometry. The complementary nature of these approaches is often best appreciated through the full breadth of physics and engineering backgrounds within the student teams. Teams quickly learn that some of their members will thrive on the computational challenges, while others will exhibit the experimental perseverance that enables the optical diagnostics.

The experimental nozzle flow apparatus used for the studies presented in this paper is described in Section 2. The threefold approach, consisting of HSV shadowgraph imaging, interferometry, and numerical simulation, is described in Section 3. Results from the experiments highlighting the complementary value of the HSV, interferometry, and numerical simulations are presented in Section 4. Finally, concluding remarks are presented in Section 5.

\section{Description of apparatus}

The nozzle flow apparatus shown in Figure 1 consists of an axisymmetric converging-diverging nozzle and a 1-gallon air pressure tank. A Mylar diaphragm is secured in a rupture disk holder that mates the nozzle to the air tank. The diaphragm ruptures at tank gage pressures of approximately $500 \mathrm{kPa}$, releasing the pressurized air through the nozzle. The nozzle is constructed with inlet, outlet, and 
throat diameters of $19.0 \mathrm{~mm}, 12.7 \mathrm{~mm}$, and $9.9 \mathrm{~mm}$, respectively. The isentropic flow relations for an ideal gas predict a Mach 2.1 exit condition for the nozzle throat to outlet area ratio.

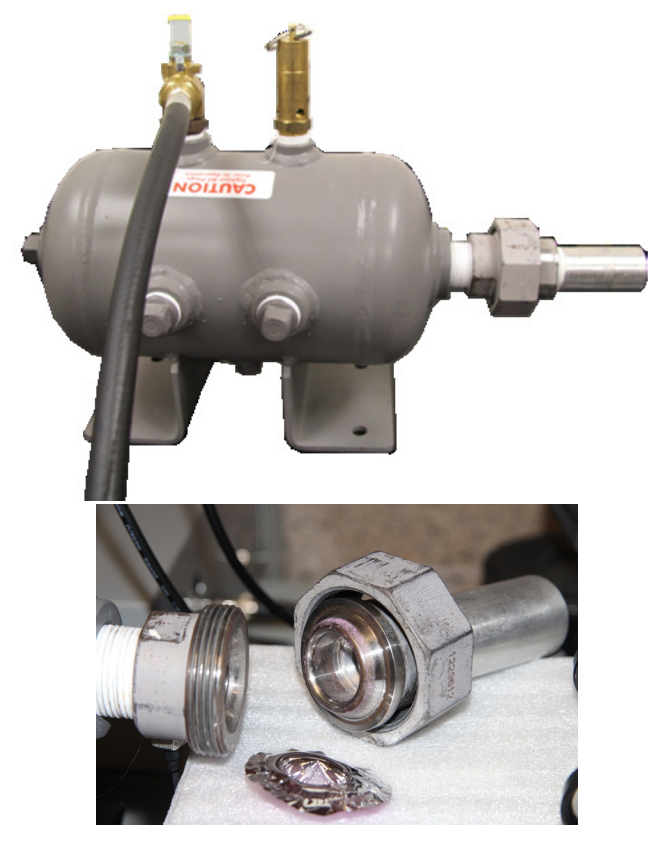

Figure 1: The nozzle apparatus (left), along with the rupture disk holder that mates the nozzle to the air tank (right).

\section{Experiments}

In this section we describe a threefold approach for characterization of the startup flow from the nozzle.

\subsection{HSV shadowgraph imaging}

Shadowgraph imaging techniques [1,2] are utilized to study the initial flow from the nozzle. The shadowgraph setup (see Figure 2) sends the light beam from a $400 \mathrm{~mW} 532 \mathrm{~nm}$ laser through an objective lens and spatial filter. The weakly expanding beam passes through the nozzle exit flow region and is projected on a frosted glass imaging window. A circular aperture is placed in the beam after the spatial filter to clean up the shadowgraph image. The shadowgraph projection on the frosted glass is recorded using a NAC Memrecam HX-3 high-speed video camera at a frame rate of 100,000 images per second and a 0.4 microsecond digital shutter. 


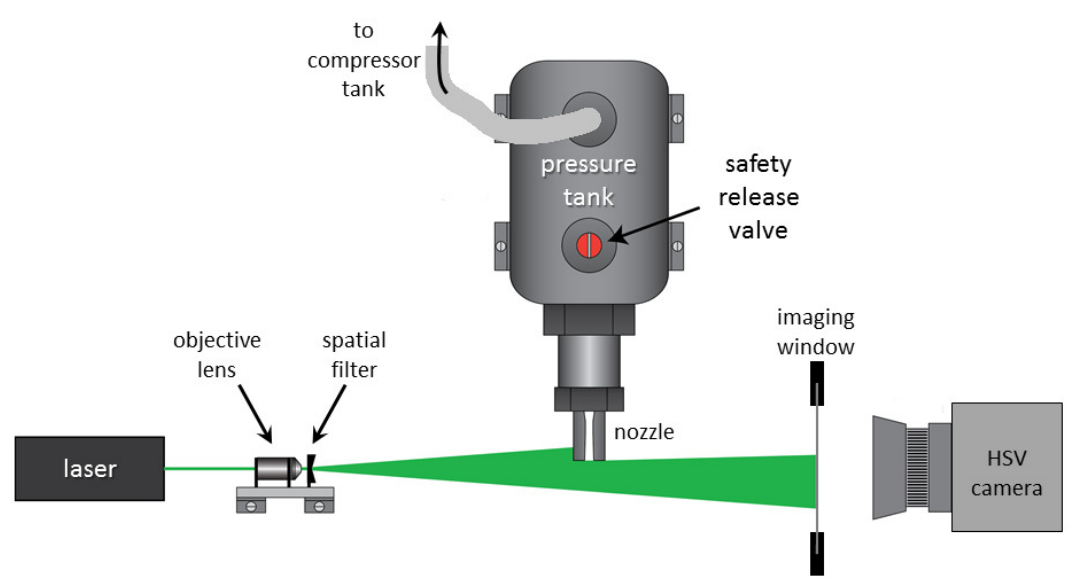

Figure 2: Shadowgraph imaging setup.

The basic optical principle in the shadowgraph imaging is that rays of light travel in straight lines, except in regions with a refractive index gradient. The altered path of the refracted light rays results in a nonuniform projection of light - or shadows - onto the frosted glass. For compressible flows, the refractive index gradient is directly related to fluid density variations in the vicinity of the nozzle flow. A linear relationship between fluid density $(\rho)$ and the refractive index $(n)$ is

$$
(n-1)=\kappa(\lambda) \cdot \rho,
$$

where $\kappa$ is the Gladstone-Dale coefficient, which slightly depends on the laser wavelength $\lambda$, and is approximately $2.25 \times 10^{-4} \mathrm{~m}^{3} / \mathrm{kg}$ for $633 \mathrm{~nm}$ light in air at standard atmospheric conditions.

The strong density gradient of the initial shock produces high-contrast shadows in the projection, whereas the supersonic flow following the shock wave produces a more gradual projection of the compressible flow features. The HSV shadowgraph recording at 100,000 frames per second provides a clear depiction of the evolution of the flow in the first 150 microseconds after the initial shock wave emerges from the nozzle (see Figure 3). The shock wave is clearly visible from $0-70 \mu$ s and is followed by the roll up of a vortex ring at the nozzle exit, along with the initial formation of Mach diamonds - standing shock waves - that remains in the nozzle exit region throughout the quasi-steady flow stage.

Although the shadowgraph image sequence provides a clear depiction of the evolution of the nozzle jet flow, the HSV sequence of the startup flow is limited to around 15 images at 100,000 frames per second. The shadowgraph technique provides an excellent qualitative depiction of the compressible flow, but is quite limited in terms of the quantitative information that the method can provide. Numerical simulation and heterodyne interferometry enhance the shadowgraph 


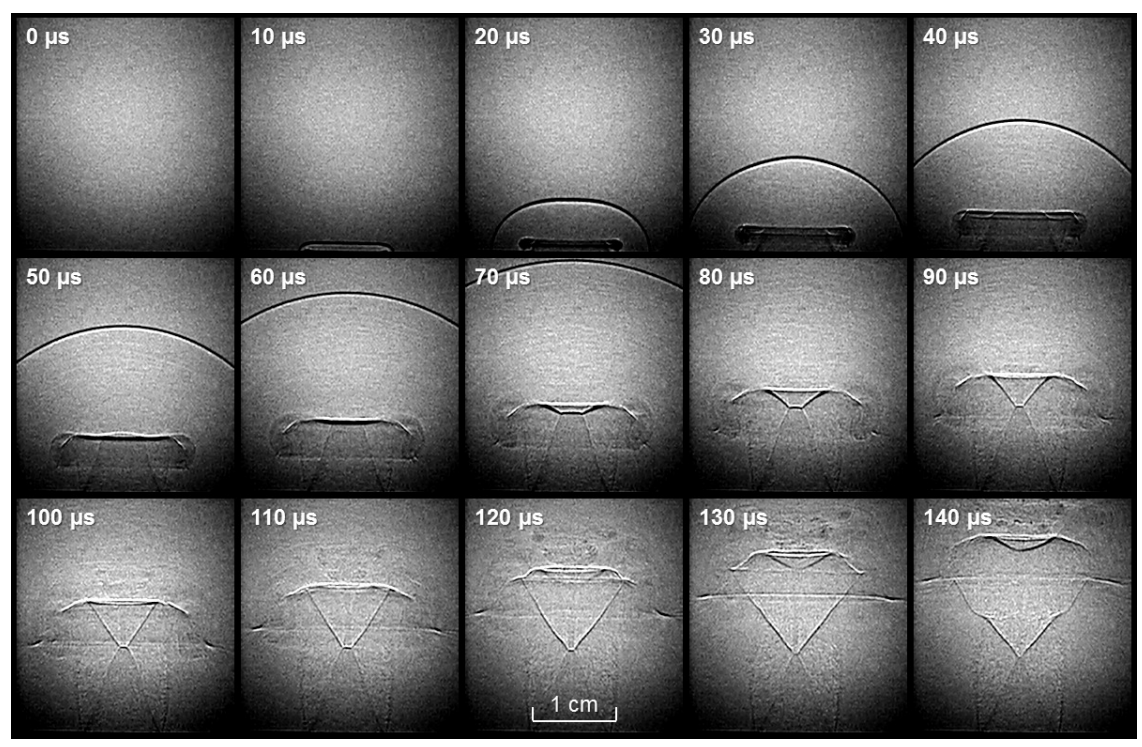

Figure 3: HSV shadowgraph image sequence portraying the initial shock wave and initial development of supersonic flow from the nozzle.

imaging by providing quantitative flow field data at much smaller time scales than for the HSV shadowgraphy.

\subsection{Numerical simulation}

Finite element computations are carried out using $\mathrm{COMSOL}^{\circledR}$ to model the unsteady, axisymmetric, compressible gas flow in a fluid domain consisting of the pressure tank, nozzle, and exit flow region. The flow in the numerical simulation is driven by an initial pressure condition, with initial pressures of $4.480 \times 10^{5} \mathrm{~Pa}$ in the tank and an atmospheric pressure of $1.013 \times 10^{5} \mathrm{~Pa}$ everywhere else. The initial pressure distribution is representative of the conditions in the physical experiments, with the diaphragm serving as the boundary between the high and low pressure regions. The fluid is prescribed to be initially at rest and at 20 degrees Celsius for the entire domain. No slip, thermally insulated boundary conditions are prescribed at all boundaries, except for along the symmetry axis. The fluid domain is discretized with an unstructured triangular mesh of approximately 200,000 elements. Finite element computations provide solutions on the discretized domain for the time-dependent, compressible flow equations for conservation of mass, momentum, and energy for an ideal gas.

The numerical simulation is carried out for sufficient simulation time to model the propagation of the shock wave and the initial formation of Mach diamonds at the nozzle exit. After the highly-transient startup flow, the nozzle establishes a Mach 2 exit condition, which is sustained during the quasi-steady flow stage. In 
this stage, gas from the tank is accelerated and becomes sonic (Mach 1) at the nozzle throat and supersonic in the diverging section of the nozzle. The gradient of the density field from the numerical simulation is displayed in Figure 4. The images depict the density gradient magnitude at $10 \mu$ s steps, with dark regions corresponding to regions of high density gradient. The images are mirrored to provide a direct comparison with the HSV shadowgraph sequence in Figure 3.

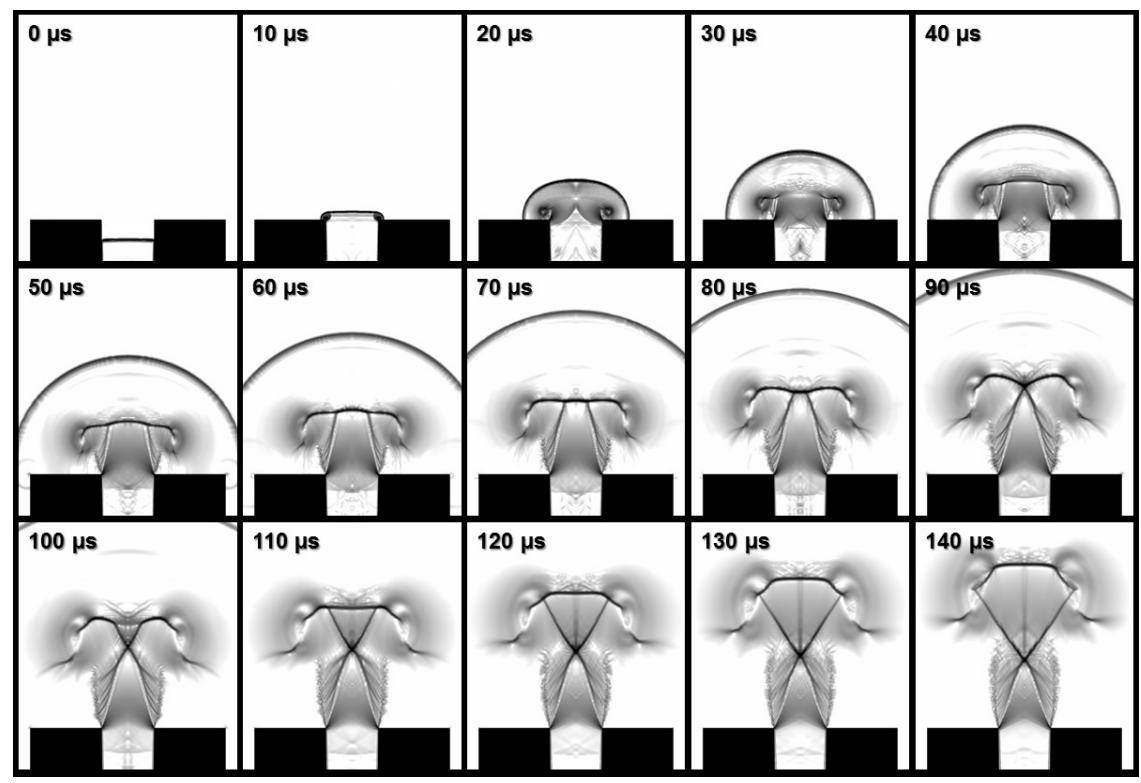

Figure 4: Numerical simulation image sequence portraying the initial shock wave and development of supersonic flow from the nozzle.

\subsection{Heterodyne interferometry}

While HSV shadowgraph imaging produces a time history of the spatial distribution of the density gradients beyond the nozzle and numerical simulation predicts the field description for the transient nozzle flow, interferometry offers an experimental method for quantitative analysis of the highly transient nozzle startup flow at desired sampling rates. This optical diagnostic tool serves to complement the computational modeling by checking the reliability of numerical predictions.

A heterodyne interferometer monitors the rapidly changing optical path length along a selected probe-line path in the nozzle vicinity. A LabVIEW ${ }^{\circledR}$ enhanced system, utilizing a stabilized $\mathrm{He}-\mathrm{Ne}$ laser at $633 \mathrm{~nm}$, measures these changes in optical path by recording phase shifts between two $80 \mathrm{MHz}$ reference signals. Such a heterodyne system has diverse applications, including surface metrology [3] and fluid shock wave diagnostics [4]. The experimental setup is shown in 
Figure 5. The beam from a frequency stabilized He-Ne laser is directed through an acousto-optic modulator (AOM), thereby frequency shifting a first-order diffracted beam by $40 \mathrm{MHz}$. The first-order beam is subsequently split into beams having orthogonal polarizations. These orthogonal beams propagate separately within the two arms of the interferometer, with one arm passing through the nozzle flow region and both arms eventually retro-reflected back along the initial path. The retro-reflected beams experience an additional $40 \mathrm{MHz}$ frequency shift upon passing again through the AOM and upon reflection from the front mirror of the laser are combined with the original zeroth order beam. The phase change data from the nozzle flow is encoded as phase shifts between the $80 \mathrm{MHz}$ beat signals that are collected by twin photo detectors. An RF mixer and LabVIEW program are then used to decode and extract the highly transient differential phase shift data between the two arms of the interferometer. Sample plots of the transient phase data and the corresponding lissajous curve are shown in Figure 6.

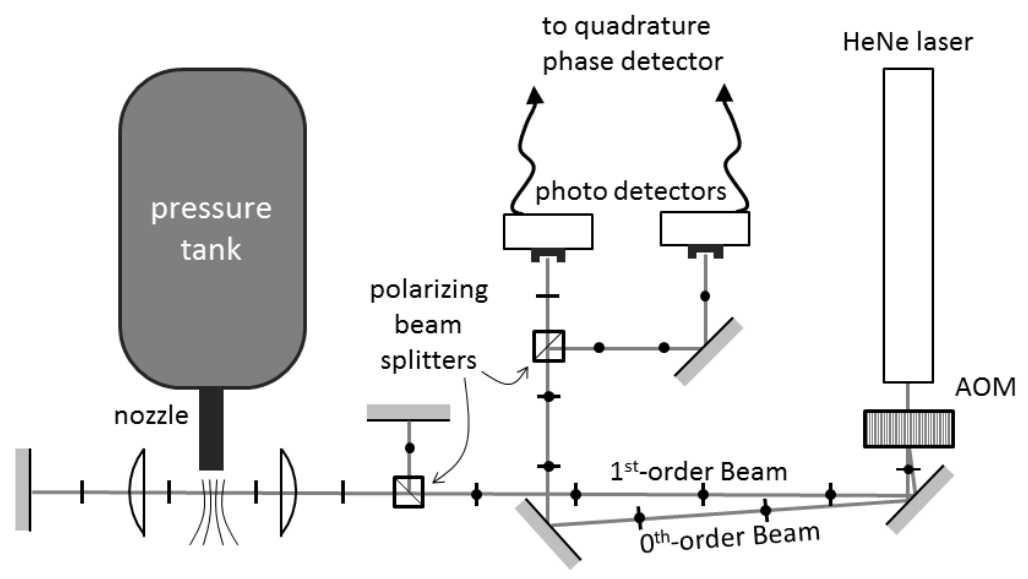

Figure 5: Heterodyne interferometer line drawing.

As the heterodyne system monitors the phase difference between the $80 \mathrm{MHz}$ beat signals, the Gladstone-Dale relationship predicts the propagation phase velocity to be proportional to the air density. Thus laser light passing through the nozzle flow experiences a phase shift relative to light passing through undisturbed air of the reference arm. This phase shift is an integrated effect due to the changing refractive index along the laser beam path through the disturbed air. As such, heterodyne measurements do not directly describe the spatial distribution of the refractive index (which allows calculation of the density field) because such information is encoded in the total phase shift observed along each probe line. However by measuring the phase shifts along a collection of distinct lines, the spatial distribution may be resolved through mathematical transformations. For the predicted axisymmetric case of the jet nozzle, this is accomplished by 

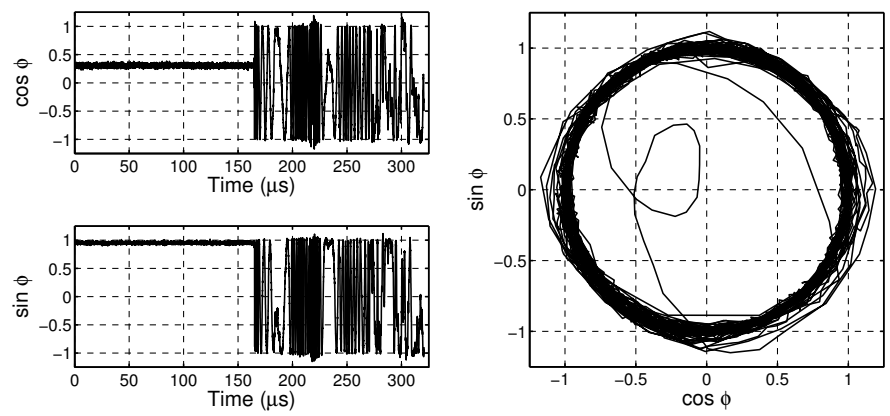

Figure 6: Heterodyne interferometer phase data: Transient phase plots (left) and the corresponding lissajous curve (right).

sampling a parallel set of lines within a plane extending perpendicular to the jet nozzle flow, and transforming them with the inverse Abel function. This allows a direct conversion of phase shifts (at $\mathrm{MHz}$ level sample rates) to density field measurements that may be compared to numerical simulation density field data.

\section{Results and analysis}

\subsection{Phase data from heterodyne interferometry and COMSOL}

Direct comparisons are made between the COMSOL simulation and heterodyne interferometer measurements on the total phase shift in the nozzle flow region. The phase shift is evaluated from the simulation with a line integral through the computed air density field.

To evaluate the integral, the incremental phase shift $(d \phi)$ over a segment of length $d y$ can be written as

$$
d \phi=2 \pi \cdot \frac{\Delta n d y}{\lambda}
$$

where $\Delta n$ represents a change in index of refraction and $\lambda$ is the laser wavelength. The total phase shift $(\phi)$ resulting from a single pass of the laser through the nozzle flow is written as

$$
\phi=\int d \phi=\frac{2 \pi \kappa(\lambda)}{\lambda} \int\left(\rho-\rho_{0}\right) d y
$$

where $\rho$ is the computed air density, $\rho_{0}$ is the ambient air density, and $\kappa(\lambda)$ is the Gladstone-Dale coefficient. The line integrals are taken along the same probe lines as sampled in the heterodyne experiment. The integrated result is doubled to account for retro-reflection of the laser beam through the probe region.

A comparison between the simulation and interferometry phase shift data is shown in Figure 7 for probe lines at positions of $y=10 \mathrm{~mm}, 15 \mathrm{~mm}$, and 
$20 \mathrm{~mm}$ downstream from the nozzle and perpendicular to the flow direction. The simulation data is given a time shift to correlate it with the interferometry data. The comparison shows good quantitative agreement in both the magnitude of the phase shifts and in the temporal spacing of the shock wave. The comparisons reveal a discrepancy in the phase shift due to the incident shock wave, with the simulation predicting a slightly greater phase shift. The simulation also predicts initial shock

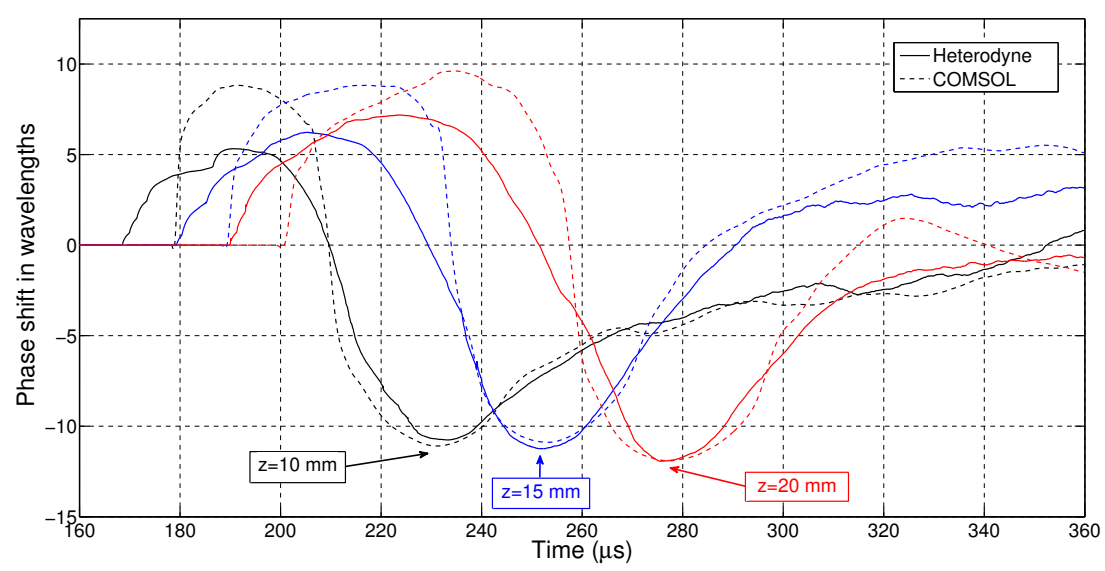

Figure 7: Total phase shift as predicted by the COMSOL simulation and measured with the heterodyne interferometer.

and compressible flow behavior that is compressed in time; with the simulated behavior about 5-10 microseconds shorter than for the interferometry data. This is likely due to the modeling of an impulsive rupture of the diaphragm, which is more abrupt than how the diaphragm bursts in the experiment. Other characteristic features, such as the depth of the trough and the magnitude of the slope rising up from it, demonstrate an excellent match between the simulation and experiment.

\subsection{Density field reconstruction based on heterodyne interferometer data}

The density field for the nozzle flow is reconstructed from heterodyne interferometer data using the Abel transform [5]. The Abel transform is applied in the $x y$-plane, which is perpendicular to the nozzle, as shown in Figure 8. From eqn (1), $n-1$ is proportional to the density of the gas. Since the heterodyne interferometer measures the change in optical path length, with the passage of time, from the initial path length of the interferometer arm through air, the signal from the interferometer will be proportional to

$$
h(y, t)=\int_{-\infty}^{\infty} f(x, y, t) d x
$$


where $f(x, y, t)$ is the change in density at a certain time due to the rupturing of the diaphragm. In what follows, the $t$ will be dropped because everything will refer to the path length and density change at a certain time.

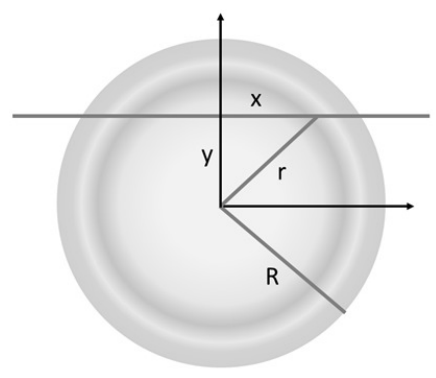

Figure 8: The Abel transform plane is located downstream from the nozzle and perpendicular to the nozzle symmetry axis.

Since $f(x, y)$ is assumed to be symmetric about the axis of the nozzle, the function and integral can be converted to

$$
h(y)=2 \int_{y}^{\infty} \frac{f(r) r}{\sqrt{r^{2}-y^{2}}} d r,
$$

which is the Abel transform of $f(r)$. Here, $r$ is the distance from the axis of the nozzle.

If measurements of $h(y)$ are made at several $y$ values, $f(r)$ can be extracted from the Abel transform. We use a MATLAB ${ }^{\circledR}$ program published by Killer [6], which is the implementation of an algorithm proposed by Pretzler [7], to perform the reconstruction of the density field. In this algorithm, it is assumed that

$$
f(r)=A_{0}+\sum_{n=1}^{u p f} A_{n} f_{n}(r),
$$

where upf stands for "upper frequency" and is a number chosen to model satisfactorily the spatial fluctuations in $f(r)$. The functions of the expansion are $f_{n}(r)=1-(-1)^{n} \cos ((n \pi r) / R)$, where $R$ is the radius of the system, the distance between $h(0)$ and $h(y \max )$. Killer's algorithm combines the $h(y)$ data with integrals of the $\{\mathrm{fn}\}$ to construct a matrix equation which can be solved for the $\{\mathrm{An}\}$, and then the $f(r)$ can be calculated. For the nozzle studies, measurements were made out to $R=2.0 \mathrm{~cm}$, using an upper frequency of $u p f=7$.

Reconstructions using the inverse-Abel transform share several key characteristics with the simulation data for the nozzle flow. The initial shock wave, seen as higher density air, is first evident near the center of the cross-sectional view after the nozzle. This section of higher density slowly moves radially outward as 
the shock wave passes further through the cross-sectional image. After the shock wave passes, the air density field also shows some similarities. The simulations and reconstructions both show oscillations between high and low air density near their centers following the initial shock wave. Due to the sensitivity of the reconstruction to data input into the Abel transform, similarities between computational and reconstructed data are inexact. However, a general semblance is evident between the two data sets, as shown in Figure 9. The density field from the COMSOL simulation is shown on the left, along with the locations of the interferometer probe lines that are perpendicular to the figure. The reconstructed density field is shown on the right, along with the same set of probe lines. Density contours are displayed for $\rho=0.5 \mathrm{~kg} / \mathrm{m}^{2}$ and $\rho=1.0 \mathrm{~kg} / \mathrm{m}^{2}$.
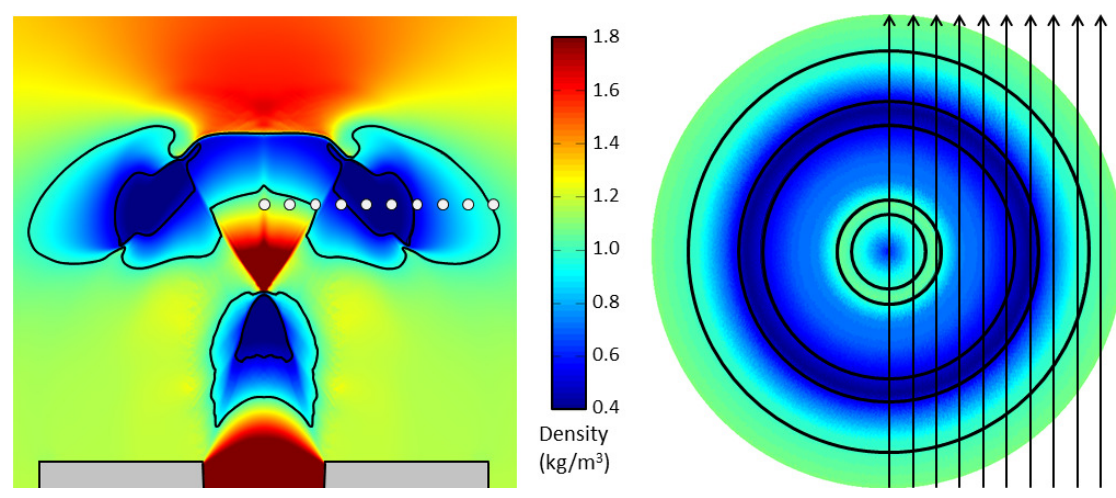

Figure 9: Abel transform: COMSOL simulation density field (left); density field reconstruction (right).

\section{Conclusion}

The threefold approach to study supersonic nozzle flow utilizes HSV, computational physics, and time-resolved interferometer gas density measurements. This strategy is pedagogically attractive for undergraduates in physics and engineering and addresses fundamental challenges for advanced undergraduate laboratories striving to provide an engaging experience that brings together ongoing research and academic studies. The complementary nature of these approaches is uniquely appreciated through diverse physics and engineering backgrounds within student teams. Teams quickly learn that some of their members will thrive on the computational challenges, while others will exhibit the experimental perseverance that enables the optical diagnostics. The threefold approach provides a richer and more comprehensive description of nozzle flow than can be achieved by the individual approaches. 


\section{Acknowledgements}

This work was supported in part by the National Science Foundation under Grant No. 1245573 and by the Minnesota Space Grant Consortium in accordance with NASA's emphasis on giving undergraduates hands-on experiences in research, especially in areas of research applicable to aeronautics and space science. We are grateful to Kent Peterson and NAC Image Technology for their support with the NAC Memrecam HX-3 camera.

\section{References}

[1] Settles, G.S., Schlieren and Shadowgraph Techniques: Visualizing Phenomena in Transparent Media. Springer-Verlag: Berlin and New York, 2001.

[2] Liepmann, H.W. \& Roshko, A., Elements of Gasdynamics. Dover: New York, 1993.

[3] Carlson, T., Groschen, R., Denzer, S., Greenlee, T., Peterson, R. \& Robinson, G., Vibration resistant direct-phase detecting optical interferometers. Applied Optics, 36(28), pp. 7162-7171, 1997.

[4] Olson, G., Peterson, R., Pulford, B., Seaberg, M., Stein, K., Stelter, C., \& Weber, R., The role of shock waves in expansion tube accelerators. American Journal of Physics, 74(12), pp. 1071-1076, 2006.

[5] Bracewell, R., The Fourier Transform and its Applications. McGraw-Hill: New York, 1965.

[6] Killer, C., Abel inverse algorithm. http://www.mathworks.com/matlab central/fileexchange/43639, 2013.

[7] Pretzler, G., A new method for numerical Abel-inversion. Zeitschrift für Naturforschung A, 46a, pp. 639-641, 1991. 